\title{
MALONDIALDEHYDE AND TOTAL ANTIOXIDANT CAPACITY AS PREDICTORS BIOMARKERS OF MORTALITY IN ACUTE ALUMINIUM PHOSPHIDE POISONING
}

\author{
BY
}

\author{
Nermin M. Emam ${ }^{1}$, Dalia Alsaied Ahmed ${ }^{1}$, Abeer Mesbah', Mostafa Mansour ${ }^{2}$ and \\ Sameera Sh. Hamed \\ ${ }^{1}$ Forensic Medicine and Clinical Toxicology Department, Faculty of Medicine, Mansoura University. ${ }^{2}$ Clinical \\ Pathology Department, Faculty of Medicine, Mansoura University, Egypt.
}

\section{ABSTRACT}

Introduction: Aluminum Phosphide "ALP" tablets are frequently used to commit suicide in developing countries and Egypt. Mortality is very high; it can induce oxidative stress and alter antioxidant defense system. Aim of the Work: This study aimed to evaluate malondialdehyde "MDA" and total antioxidant capacity "TAC" as oxidative stress biomarkers in acute ALP poisoning and correlated them with poisoned patients' outcome and mortality. Subjects and Methods: This study was conducted on 120 individuals of both sexes divided into; (Group I) included 60 acute ALP poisoning patients, presented within 6 hours of exposure to Toxicology Unit of Emergency Hospital, Mansoura University and the remaining 60 (Group II) were healthy individuals as control group. Results: Serum level of MDA was significantly increased and TAC was significantly reduced in ALP poisoned group at time of presentation in comparison to control group. On the other hand, levels of MDA and TAC were statistically significant difference between survivors and expired groups. In addition, serum MDA and TAC were found to be sensitive in prediction of ALP mortality. However, MDA level showed maximum specificity as well as more accuracy for prediction of poor outcomes. Conclusions: It can be concluded that, ALP exerts its toxicity through developing disproportionate oxidative stress and compromising antioxidant protective mechanisms. Both MDA and TAC were sensitive in prediction of ALP mortality and serum MDA level could be used as a more sensitive specific predictor biomarker for poor prognosis in acute ALP poisoning.

Keywords: Aluminum Phosphide, Oxidative Stress, Malondialdehyde, Total Antioxidant Capacity, Mortality.

Corresponding author: Dr. Nermin M. Emam

Email: nermin_toxo1981@hotmail.com

\section{INTRODUCTION}

$A$ cute aluminum phosphide (ALP) developing countries and Egypt over the past few years. It is a solid fumigant pesticide that is used to keep rats and other insects out of stored grain. The widespread use of ALP is attributed to be highly effective, does not affect seed viability, cheap and leaving no toxic residues in the environment. Rice tablet is the most common form of ALP (Farahani et al., 2016).

Because of its low cost and widespread availability, it has become one of the leading causes of suicide in many countries. Since the first available report of ALP poisoning in the early 1980s from India, it is now one of the most common causes of poisoning among agricultural pesticides. Most of the case series are reported from India, while some others are reported from Iran, Sri Lanka, and Morocco, with case reports from many developed countries (Gurjar et al., 2011). In Egypt, ALP tablets are frequently used to commit suicide especially among teenagers and constituting a frequent cause of admission to poison control centers (Badawi et al., 2018).

Mortality is remarkably high due to immediate release of lethal phosphine gas $\left(\mathrm{PH}_{3}\right)$ when it encounters either ambient moisture or stomach hydrochloric acid. Phosphine gas is immediately absorbed through lungs and stomach causing intoxication (Hashemi-Domeneh et al., 2016). Toxic manifestations usually develop within few minutes in the form of nausea, vomiting, diarrhea, and retrosternal burning 
pain, followed by cardiovascular, respiratory, and nervous systems manifestations. Later, hepatic failure, renal failure and disseminated intravascular coagulation may also occur (Agrawal et al., 2015).

Various mechanisms for phosphide toxicity have been identified. One of the most significant suggested mechanisms is oxidative stress induction with generation of reactive oxygen species (ROS) that are extremely harmful to biological macromolecules causing lipid peroxidation, cell membrane protein denaturation and eventually contribute to cell death (Nath et al., 2011 and Mehrpour et al., 2012).

The imbalance between the development of free radicals and the body's antioxidant protection is known as oxidative stress. Reactive oxygen species cause lipid peroxidation that degrade polyunsaturated lipids, forming malondialdehyde (MDA) that is used as a biomarker to determine an organism's level of oxidative stress (Ayala et al., 2014).

On the other hand, for all researchers interested in the role of free radical damage in diseases, the precise evaluation of oxidative stress and antioxidants' amount in biological systems is considered a challenge. The antioxidant body defense includes enzymes such as catalase, glutathione peroxidase and superoxide dismutase. Also, macromolecules such as ceruloplasmin, ferritin and albumin, in addition to small molecules such as alphatocopherol, ascorbic acid, reduced glutathione, beta-carotene, uric acid and bilirubin are considered body antioxidants (Khan et al., 2018).

Different antioxidants vary in their reducing power. Compared to that obtained by measuring individual components, the overall antioxidant activity provides more relevant biological details. It reflects the overall impact of all antioxidants which are found in plasma and body fluids. The total antioxidant capacity (TAC) of the system is equal to the amount of endogenous antioxidants (Rani and Mythili, 2014). The main advantage of this test is that it determines the antioxidant potential of all antioxidants in a biological sample, rather than just one compound's antioxidant efficiency (Kariman et al., 2012).

Aim of The Work

The aim of the present work was to evaluate levels of MDA and the TAC in acute ALP poisoning as oxidative stress biomarkers and to correlate them with poisoned patients' outcome and mortality.

○ Study Design:

SUBJECTS AND METHODS

A case control study was conducted on 120 individuals of both sexes, in the period between November 2018 to December 2019. Candidates in the present study were divided into two groups:

I. Group I: included 60 acute ALP poisoned patients presented within 6 hours of exposure to the Emergency Hospital Toxicology Unit, Mansoura University, Egypt.

II. Group II (Control group): included 60 healthy subjects for estimation of the average range of oxidative stress markers among Egyptian population.

The acceptance from the "Institutional Review Board" (IRB), Faculty of Medicine, Mansoura University had been got with code number (R/18.09.282). Informed written consent was taken from all participants or his/her guardian "if the patient was unable to participate in consent process". During the study period, after collecting detailed information about each poisoned patient, the data was kept confidential by assigning each patient a code number.

- Inclusion criteria:

Subjects (aged 12 years or older) with symptomatic acute ALP poisoning and a history of phosphide tablets ingestion presented within 6 hours from exposure with no previous medical management in any hospital or health care unit prior to admission were included in the study.

- Exclusion criteria:

Subjects less than 12 years of age, nonmanifested subjects or with doubtful ingestion, 
cases of multiple concomitant poisoning, patients presenting more than 6 hours of intake the toxic rice tablets, patients treated in any medical center before admission, and those who refused to give consent were all excluded. Pregnant and lactating women, patients with a history of diabetes mellitus, chronic heart, respiratory, kidney or liver diseases were also excluded from the study.

- Sampling:

Blood samples were taken from all patients on admission before administration of any medication. Five $\mathrm{ml}$ of venous blood samples were collected in plain vacutainer test tubes under full aseptic conditions. The serum samples were then separated by centrifugation at $3000 \mathrm{rpm}$ for 10 minutes and frozen at ($20^{\circ} \mathrm{C}$ ). Samples brought to room temperature when performing the assay.

\section{- Biochemical studies:}

Every subject's oxidative stress state was determined by measuring serum MDA and TAC levels as oxidative stress biomarkers using commercial kits supplied by Biodiagnostic, Egypt. As a lipid peroxidation product, MDA developed a complex called "Thiobarbituric acid reactive substance" (TBARS) with TBA (2-thiobarbituric acid) that was spectrophotometrically determined. Samples of MDA were analyzed sequentially after calibration of the photometer. The proteins were first precipitated using "sulphuric and phosphotungstic acid" in $0.15 \mathrm{~mL}$ serum. Precipitate that obtained was incubated with "TBA" in a "water bath at $95^{\circ} \mathrm{C}$ for 60 minutes" in an oxygen rich environment and " $\mathrm{pH}=3.4$ ". The TEP (1,1,3,3tetraethoxypropane) was used as MDA standard and "Thiobarbituric acid reactivity" was calculated as MDA "micromole per liter" ( $\mu \mathrm{mol} / \mathrm{L})$. Finally, using "Shimadzu UV-1201V spectrophotometry" at "532 nm", the colored "TBARS" complex [MDA-(TBA)2] was measured (Yagi, 1984).

Samples of TAC were analyzed in one setting after calibration of the photometer. To assess antioxidant activity, the antioxidant reaction in the sample with a specific amount of exogenously supplied hydrogen peroxide " $\mathrm{H}_{2} \mathrm{O}_{2}$ " is measured. A certain amount of the given substrate " $\mathrm{H}_{2} \mathrm{O}_{2}$ " is removed by the antioxidants in the sample then the residual " $\mathrm{H}_{2} \mathrm{O}_{2}$ " was colorimetrically determined. First the substrate " $\mathrm{H}_{2} \mathrm{O}_{2}$ " was diluted 1000 times before use, then equal amounts of chromogen and the enzyme-buffer were mixed as "working reagent". The substrate " $\mathrm{H}_{2} \mathrm{O}_{2}$ " was added to 0.5 $\mathrm{ml}$ of blank and sample and then mixed well and incubated 10 minutes at $37^{\circ} \mathrm{C}$ then the "working reagent" was added to both, mixed well, and incubated 5 minutes at $37^{\circ} \mathrm{C}$. The "absorbances of blank $\left(\mathrm{A}_{\mathrm{B}}\right)$ and sample $\left(\mathrm{A}_{\mathrm{SA}}\right)$ " were immediately read against water at "505 $\mathrm{nm}$ " and then the TAC was calculated in millimole per liter " $\mathrm{mmol} / \mathrm{L}$ " using this equation "TAC $=\left(\mathrm{A}_{\mathrm{B}}-\mathrm{A}_{\mathrm{SA}}\right) \times$ 3.33". Low TAC is considered an indicative of exposure to oxidative stress and increased susceptibility to oxidative damage (Koracevic et al., 2001).

\section{- Study Enrollment Procedures:}

All patients were subjected to detailed history taking focusing on age, gender, residence, occupation, manner and cause of poisoning, amount and route of exposure, time elapsed before the admission into hospital and the history of medical diseases. Diagnosis was confirmed by history, its specific odour, typical clinical manifestations that occurred shortly after exposure with reliable identification of the compound brought by patient attendants. General clinical examination, monitoring of vital signs and oxygen saturation were done. Additionally, assessment of level of consciousness by Glasgow coma scale (GCS) and cardiovascular examination including Electrocardiography (ECG) were done with regular measurement and recording of their vital signs, oxygen saturation, and conscious level. Shock was diagnosed if mean arterial pressure $(\mathrm{MAP})<60 \mathrm{~mm} \mathrm{Hg}$ or a systolic blood pressure $<90 \mathrm{~mm} \mathrm{Hg}$ with low urine output (Leone et al., 2015). Immediately after admission arterial blood samples were obtained 
for blood gas analysis. During the study, all information were kept confidentially.

\section{○ Outcome Measures}

All patients were followed until they were discharged from the hospital or died. The primary outcome was categorized as survivor and non-survivor.

- Statistical analysis

The "Statistical Package of Social Science (SPSS) program" for Windows "Standard Version 24" was used to analyze the results. The normality of the data was first tested with the "Kolmogorov-Smirnov test" single sample. Numbers and percentages were used to define qualitative results. The "Chisquare test" was used to for the relation between categorical variables, while the "Fischer exact test" was used when the predicted cell count was less than 5. The "Student t test" for parametric data and the "Mann Whitney test" for non-parametric data were used to compare between the two groups. The receiver operating characteristic curve "ROC curve" was used to evaluate the sensitivity and specificity at various cut-off points. At values of " $\leq 0.05$ and $\leq 0.001$," the P-value was considered significant and highly significant, respectively.

\section{RESULTS}

During the study period, a total of 120 individuals of both sexes divided into 60 acute ALP poisoned patients (Group I) presented to "Toxicology Unit of Emergency Hospital Mansoura University" and 60 healthy control subjects (Group II). Regarding socioeconomic data, the mean age of ALP poisoned patients was $24.42 \pm 10.03$ years and it was $23.47 \pm 8.35$ years in control one. Most of the studied cases in (Group I) were females 38 (63.3\%), students $30(50 \%)$ and from rural areas $49(81.7 \%)$ with no statistically significant differences between Group I and Group II regarding age, sex, residence, and occupation as shown in (Table 1).

Regarding ALP poisoned patients' characteristics (Table 2), all cases in the present study were suicidal manner of poisoning, 51 cases $(85 \%)$ due to family troubles and 9 cases (15\%) were due to financial causes. On admission, more than half of the studied patients $33(55 \%)$ presented with no garlic odour, 50 cases $(83.3 \%)$ had ingested from half to one tablet with average ingestion $0.5(0.25-2.0)$ tablet in all poisoned cases. The average time lag from ingestion of ALP tablets to medical attention was $1.22 \pm 0.59 \mathrm{hr}$. All ALP poisoned patients were presented with gastrointestinal manifestations in the form of vomiting and abdominal pain. Diarrhea occurred in 23 cases (38.3\%) and haematemesis occurred only in $3(5 \%)$ of all poisoned patients. About 7 cases (11.7\%) presented with GCS 14, the mean pulse rate was $104.57 \pm 20.51 / \mathrm{min}$ with 33 cases (55\%) presented with sinus tachycardia. The mean systolic blood pressure was 92.16 \pm 21.95 $\mathrm{mmHg}$ and diastolic blood pressure was $56.33 \pm 16.00 \mathrm{mmHg}$ with 28 cases $(46.7 \%)$ presented with shock. The mean respiratory rate was $24.66 \pm 5.50 / \mathrm{min}$ with 41 cases (68\%) presented with tachypnea, 18 cases $(30 \%)$ with hypoxemia $\left(\mathrm{SpO}_{2}<95 \%\right)$ and 36 cases $(60 \%)$ with metabolic acidosis [PH 6.9-7.34]. The mortality rate in the present study was about 19 cases $(31.7 \%)$.

Regarding oxidative stress biomarkers, there were high statistically significant differences in the levels of MDA and TAC between all ALP poisoned patients (Group I) and healthy control group (Group II) $(\mathrm{P} \leq 0.001)$ as shown in (Table 3) and (Figure 1, 2).

On the other hand, levels of MDA and TAC were statistically significantly different among survivors when compared with those who expired $\left(\mathrm{p} \leq 0.001^{*}\right)$ as shown in (Table 4) and (Figures 3, 4).

On evaluating the "ROC curve" for prediction of mortality using MDA and TAC biomarkers (Table 5), the area under curve "AUC" for MDA at the time of admission was 1.0 and the cutoff value was $68.0 \mu \mathrm{mol} / \mathrm{L}$ which regressed to have $100 \%$ sensitivity and $97.6 \%$ specificity with $98.3 \%$ accuracy for prediction of ALP poisoning mortality (Figure 5). On the 
other hand, AUC for TAC at the time of admission was 0.99 and the cutoff value was $0.45 \mathrm{mmol} / \mathrm{L}$ which regressed to have $100 \%$ sensitivity and $92.7 \%$ specificity with $95 \%$ accuracy for prediction of ALP poisoning mortality (Figure 6).

Table (1): Comparison between ALP poisoned patients and healthy control group regarding sociodemographic data.

\begin{tabular}{|c|c|c|c|c|}
\hline Variables & $\begin{array}{l}\text { ALP Poisoned } \\
\text { Group I } \\
(\mathrm{n}=60)\end{array}$ & $\begin{array}{c}\text { Healthy Control } \\
\text { Group II } \\
(\mathrm{n}=60)\end{array}$ & $\begin{array}{c}\text { Test of } \\
\text { significance }\end{array}$ & $\mathrm{P}$ value \\
\hline $\begin{array}{l}\text { Age "years" } \\
\text { Mean } \pm \text { SD }\end{array}$ & $24.42 \pm 10.03$ & $23.47 \pm 8.35$ & $t=0.564$ & 0.574 \\
\hline $\begin{array}{l}\text { Sex } \\
\text { Male } \\
\text { Female }\end{array}$ & $\begin{array}{l}22(36.7 \%) \\
38(63.3 \%)\end{array}$ & $\begin{array}{l}28(46.7 \%) \\
32(53.3 \%)\end{array}$ & $\chi^{2}=1.23$ & 0.267 \\
\hline $\begin{array}{l}\text { Residence } \\
\text { Rural } \\
\text { Urban }\end{array}$ & $\begin{array}{l}49(81.7 \%) \\
11(18.3 \%)\end{array}$ & $\begin{array}{l}50(83.3 \%) \\
10(16.7 \%)\end{array}$ & FET & 1.00 \\
\hline $\begin{array}{l}\text { Occupation } \\
\text { Student } \\
\text { Housewife } \\
\text { Farmer } \\
\text { Manual worker }\end{array}$ & $\begin{array}{c}30(50 \%) \\
9(15 \%) \\
15(25 \%) \\
6(10 \%)\end{array}$ & $\begin{array}{c}26(43.3 \%) \\
14(23.3 \%) \\
11(18.3 \%) \\
9(15 \%)\end{array}$ & $\chi^{2}=2.59$ & 0.460 \\
\hline
\end{tabular}

n: number, SD: standard deviation, $t:$ student $t$-test, $\chi^{2}$ : Chi square test, FET: Fischer exact test, $P$ is significant if $\leq 0.05$.

Table (2): ALP poisoned patients' characteristics:

\begin{tabular}{ll}
\hline \multicolumn{1}{c}{ Variables } & $\begin{array}{c}\text { ALP Poisoned Group } \\
(\mathrm{n}=60)\end{array}$ \\
\hline Family History & $51(85 \%)$ \\
Family troubles & $9(15 \%)$ \\
Financial & $33(55 \%)$ \\
\hline Odour & $27(45 \%)$ \\
No smell & \\
Garlic & $0.5(0.25-2.0)$ \\
\hline Number of tablets ingested & $3(5 \%)$ \\
Median (min-max) & $23(38.3 \%)$ \\
2 tablets & $27(45 \%)$ \\
1 tablet & $7(11.7 \%)$ \\
$1 / 2$ tablet & $1.22 \pm 0.59$ \\
$1 / 4$ tablet & $34(56.7 \%)$ \\
\hline Time lag from time of ingestion to medical attention (hour) & $23(38.3 \%)$ \\
\hline Presenting complaints (symptoms) & $3(5 \%)$ \\
Abdominal pain - Vomiting & $7(11.7 \%)$ \\
Abdominal pain - Vomiting - Diarrhea & $33(55 \%)$ \\
Abdominal pain - Vomiting - Haematemesis & $28(46.7 \%)$ \\
\hline GCS <15 & $41(68.3 \%)$ \\
\hline Sinus tachycardia & $18(30 \%)$ \\
\hline Tachypnea & $36(60 \%)$ \\
\hline Hypoxemia (SpO $2<95 \%)$ & $41(68.3 \%)$ \\
\hline Metabolic acidosis & $19(31.7 \%)$ \\
\hline Mortality rate & $25 a t u r a t 0 n$
\end{tabular}

n: number, min: minimum, max: maximum, GCS: Glasgow coma score, $\mathrm{SpO}_{2}: \mathrm{O}_{2}$ saturation. 
Table (3): Comparison of the median levels of oxidative stress biomarkers MDA and TAC between ALP poisoned patients and healthy control group:

\begin{tabular}{lcccc}
\hline Variables & $\begin{array}{c}\text { ALP Poisoned Group } \\
\mathrm{I} \\
(\mathrm{n}=60)\end{array}$ & $\begin{array}{c}\text { Healthy Control } \\
\text { Group II } \\
(\mathrm{n}=60)\end{array}$ & $\begin{array}{c}\text { Test of } \\
\text { significance }\end{array}$ & P value \\
\hline $\begin{array}{l}\text { MDA } \mu \text { mol/L median } \\
(\min -\mathrm{max})\end{array}$ & $\begin{array}{c}47.00 \\
(34-266)\end{array}$ & $\begin{array}{c}11.50 \\
(7-18)\end{array}$ & $\mathrm{Z}=7.71$ & $\leq 0.001^{*}$ \\
\hline $\begin{array}{l}\text { TAC mmol/L median } \\
(\min -\mathrm{max})\end{array}$ & $\begin{array}{c}0.70 \\
(0.10-1.70)\end{array}$ & $\begin{array}{c}3.9 \\
(2.7-4.9)\end{array}$ & $\mathrm{Z}=7.75$ & $\leq 0.001^{*}$ \\
\hline
\end{tabular}

n: number, MDA: malondialdehyde, TCA: Total antioxidant capacity, $\mu m o l / L:$ micromole per liter, mmol/L: millimole per liter, min: minimum, max: maximum, Z: Mann Whitney $U$ test. $P$ is significant if $\leq 0.05$ and highly significant if $\leq 0.001$.

Table (4): Comparison of oxidative stress biomarkers MDA and TAC between survived and expired groups:

\begin{tabular}{lcccc}
\hline Variables & Survived & Died & $\begin{array}{c}\text { Test of } \\
\text { significance }\end{array}$ & p-value \\
\hline $\begin{array}{l}\text { MDA } \mu \text { mol/L median } \\
(\min -m a x)\end{array}$ & $\begin{array}{c}42 \\
(34-73)\end{array}$ & $\begin{array}{c}163 \\
(120-266)\end{array}$ & $\mathrm{Z}=6.19$ & $\leq 0.001^{*}$ \\
\hline $\begin{array}{l}\text { TAC mmol/L median } \\
(\min -\max )\end{array}$ & 0.9 & 0.2 & $\mathrm{Z}=6.14$ & $\leq 0.001^{*}$ \\
\hline
\end{tabular}

MDA: malondialdehyde, TCA: Total antioxidant capacity, $\mu \mathrm{mol} / \mathrm{L}:$ micromole per liter, mmol/L: millimole per liter, min: minimum, max: maximum, $Z$ : Mann Whitney $U$ test. $P$ is significant if $\leq 0.05$ and highly significant if $\leq 0.001$.

Table (5): Receiver operating characteristic curve "ROC" for prediction of mortality using MDA and TAC oxidative stress biomarkers:

\begin{tabular}{cccccccccc}
\hline \multirow{2}{*}{ Variables } & \multirow{2}{*}{ AUC } & \multicolumn{2}{c}{$\mathbf{9 5 \%}$ CI } & Cutoff & Sensitivity & Specificity & PPV & NPV & Accuracy \\
\cline { 3 - 6 } & & Lower & Upper & & & & & & \\
\hline MDA & 1.0 & 1.0 & 1.0 & 68.0 & $100 \%$ & $97.6 \%$ & 95 & 100 & $98.3 \%$ \\
\hline TAC & 0.99 & 0.98 & 1.0 & 0.45 & $100 \%$ & $92.7 \%$ & 86.4 & 100 & $95 \%$ \\
\hline
\end{tabular}

MDA: malondialdehyde, TCA: Total antioxidant capacity, AUC: area under the curve, CI: confidence interval, PPV: positive predictive value, NPV: negative predictive value. 


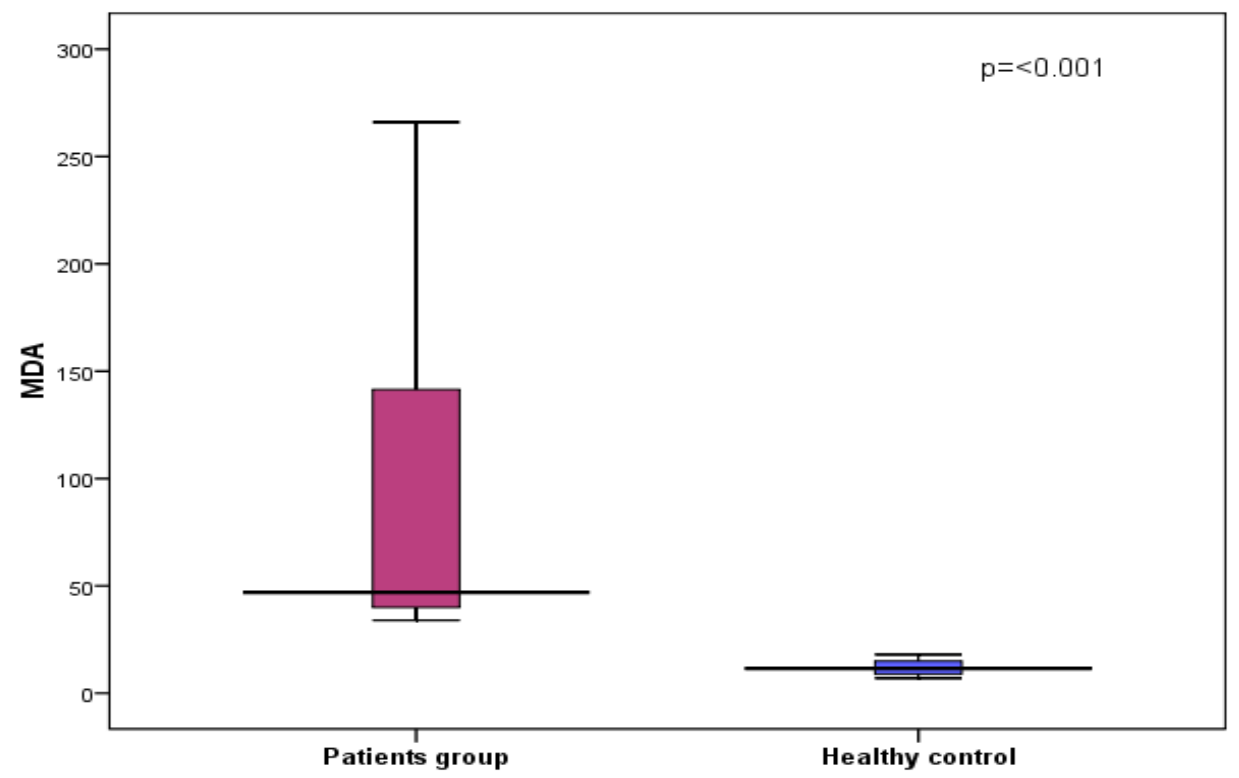

Figure (1): Box plot for median MDA level among the studied groups.

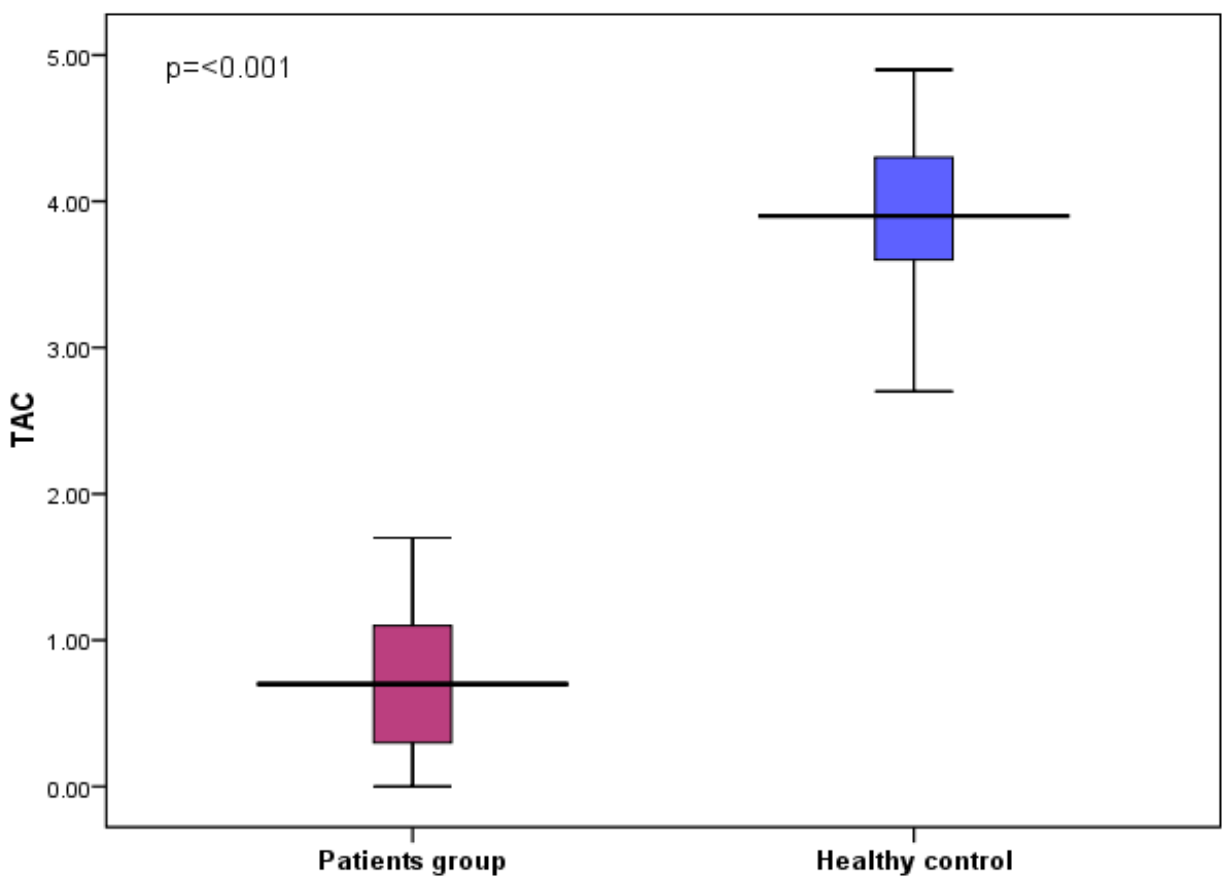

Figure (2): Box plot for median TAC level among the studied groups. 


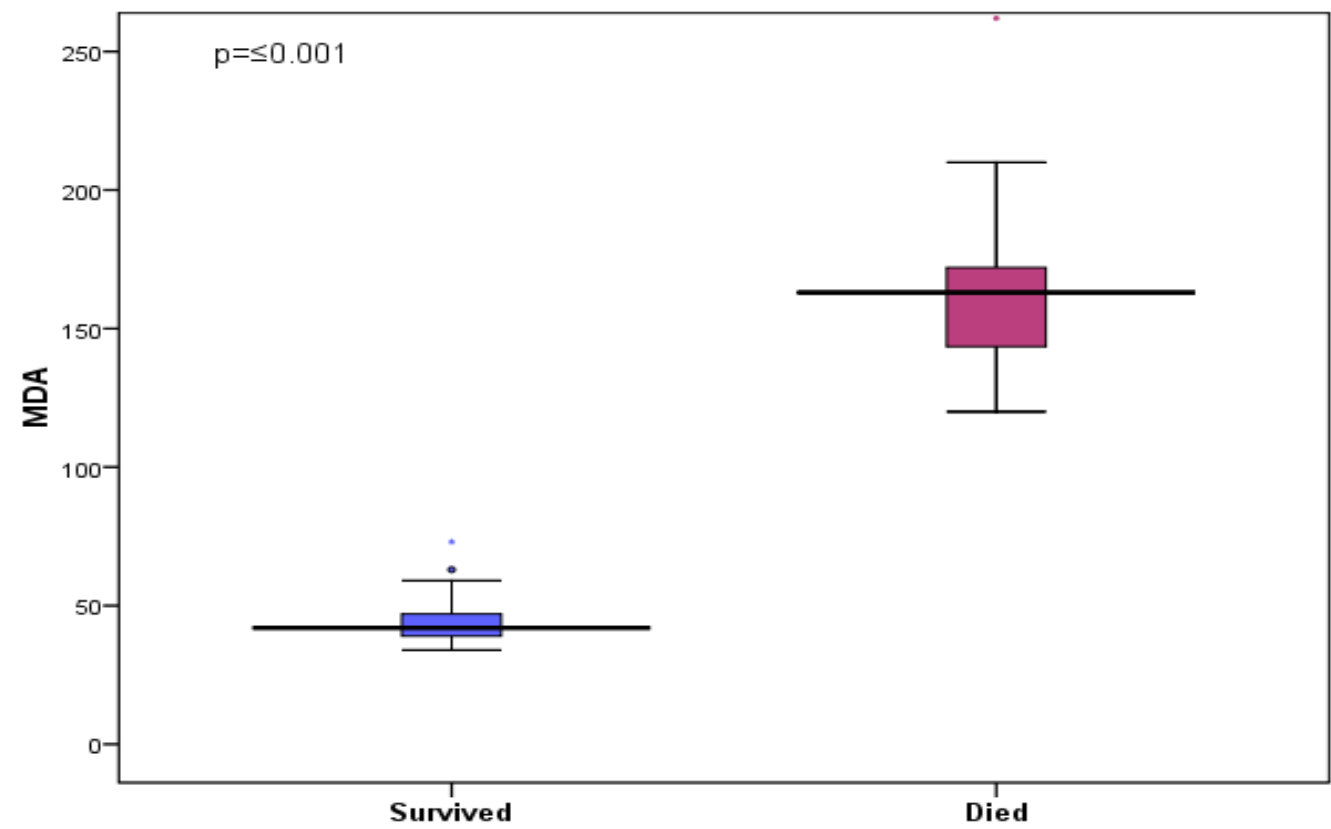

Figure (3): Box plot for median MDA level among survived and died patients.

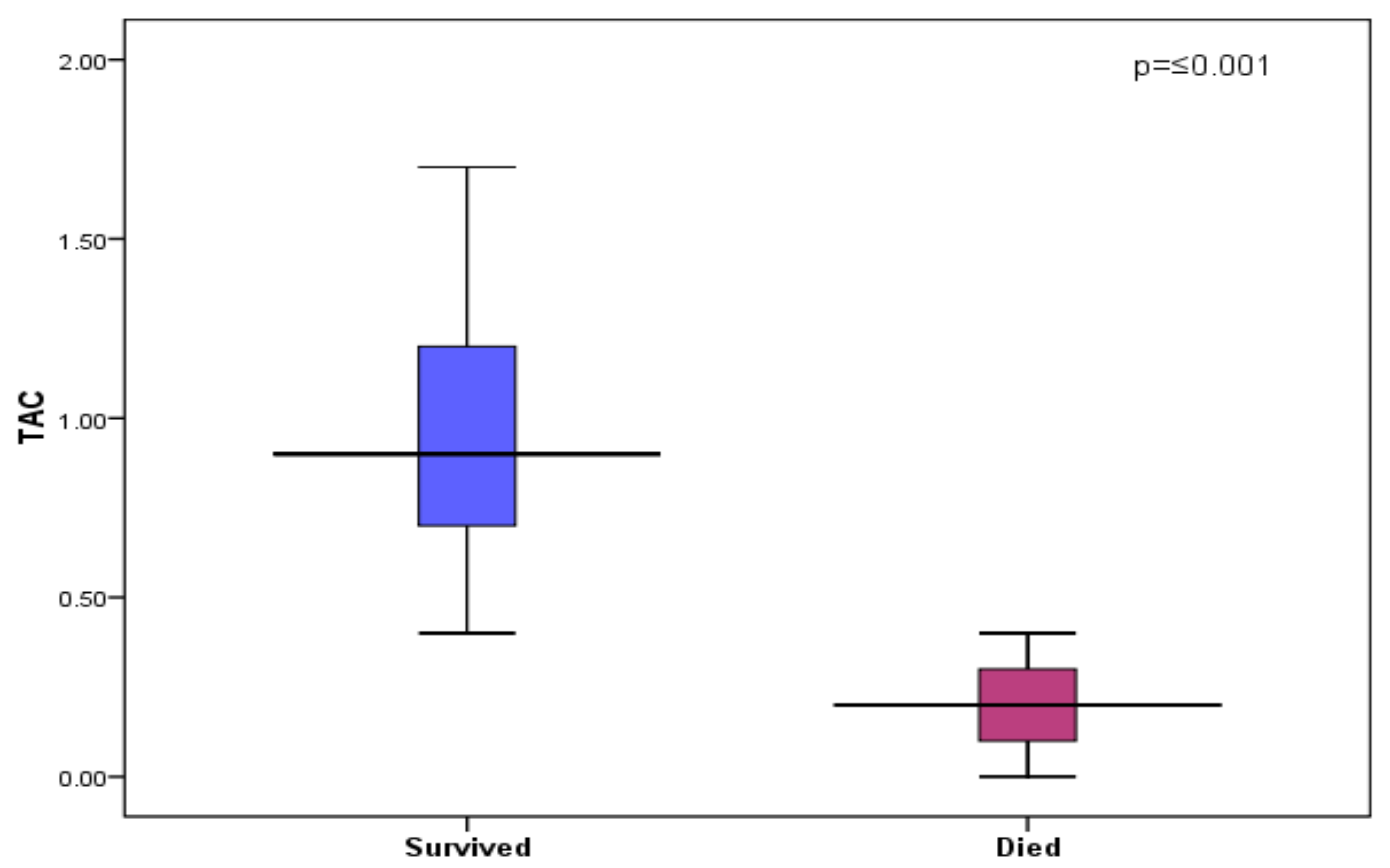

Figure (4): Box plot for median TAC level among survived and died patients. 
ROC Curve

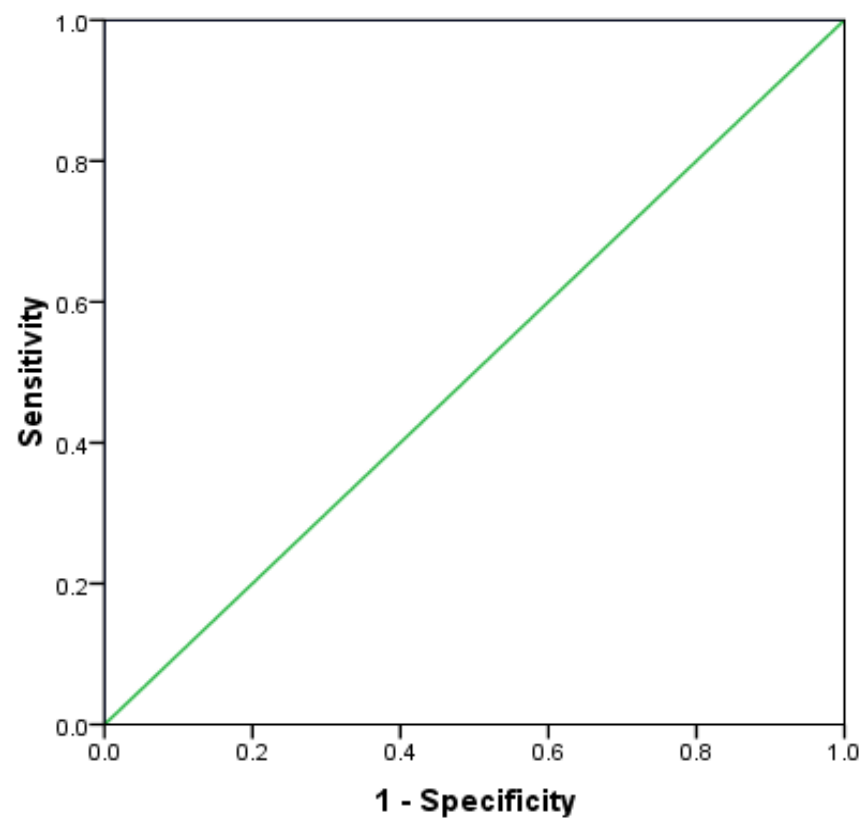

Figure (5): ROC curve for prediction of mortality using MDA biomarker.

ROC Curve

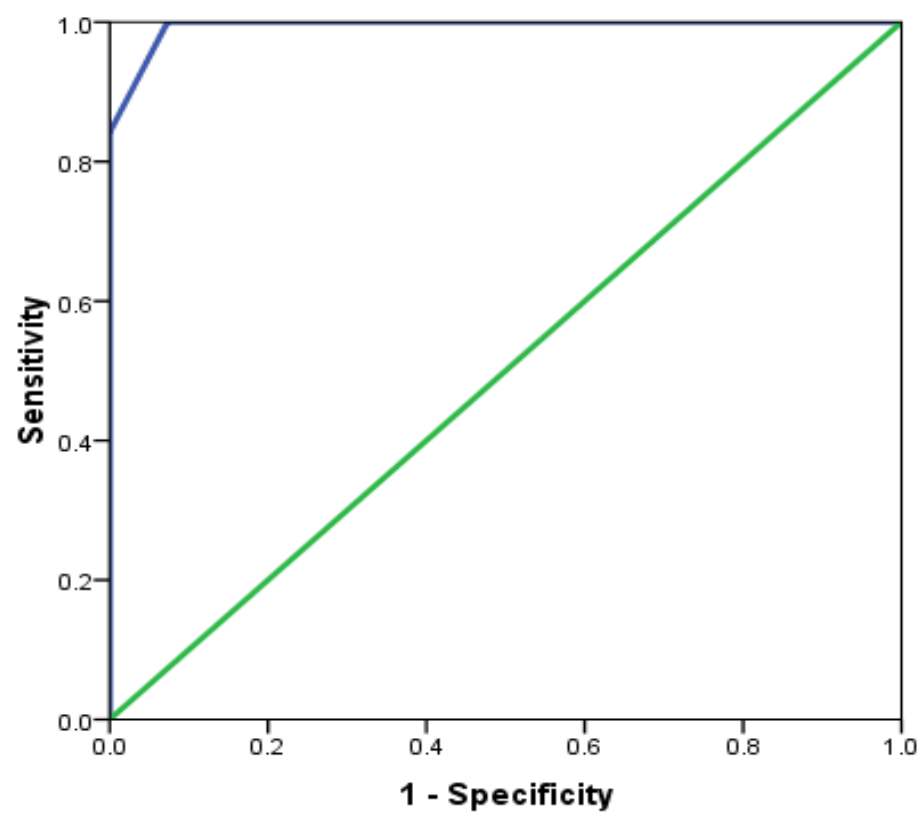

Figure (6): ROC curve for prediction of mortality using TAC biomarker. 


\section{DISCUSSION}

Aluminum phosphide is a low-cost solid fumigant that is widely used to preserve grains and is considered a highly toxic pesticide. During the past four decades, ALP has shown an increased misuse in order to commit suicide (Singh et al., 2014). ALP poisoning is an extremely lethal poisoning, having a high mortality rate among humans. Severe ALP poisoning has the potential to induce multi organ failure (Oghabian and Mehrpour, 2016). According to previous animal studies, ALP can trigger oxidative stress by causing free radical generation and altering the antioxidant protection mechanism. A similar mechanism can also play a role in human ALP toxicity pathogenesis and lead to the missing link (Kariman et al., 2012).

The aim of this study was to determine the levels of oxidative stress biomarkers; MDA as a product of lipid peroxidation and the level of TAC as an indicative of oxidative stress in acute ALP poisoning and to correlate these oxidative stress biomarkers with the poisoned patients' outcome.

Regarding sociodemographic data (Table 1), both ALP poisoned patients and healthy control group were similar that is to reduce the study's bias. The mean age of ALP poisoned patients was $24.42 \pm 10.03$ years. Most of the ALP poisoned patients were young, came from rural areas and predominantly female students. That may be due to its low price and easy accessibility of ALP tablets as well as the psychological liability of the females. Sheta et al., (2019) concluded also that females outnumbered males in all acute ALP poisoning cases with a sex ratio of $2.75: 1$. In contrast, in a study conducted by Mathai and Bhanu (2010), it was observed that the majority of patients were young males due to their work in agriculture fields and storage of grain where it is widely used.

Regarding poisoned patients' characteristics (Table 2), all cases were suicidal manner of poisoning due to family troubles that was reported also by Gunnell et al., (2007); the most common method of ALP poisoning is self-poisoning. There was no garlic odour in $55 \%$ of poisoned cases. Bogle et al., (2006) had observed that, there was no characteristic garlic smell of phosphine in their studied cases also.

Most of the ALP poisoned cases (83.3\%), had ingested from $1 / 2$ to 1 recently opened rice tablet with average ingestion 0.5 (0.25-2.0) tablet in all poisoned cases. The average time lag between ingestion and medical attention was about $1.22 \pm 0.59 \mathrm{~h}$. and the mortality rate in the present study was about $31.7 \%$. According to Bajpai (2010), the mortality rates for ALP poisoning reported in the literature range from $37 \%$ to $80 \%$. On the other hand, Meena et al., (2015) had reported that the overall fatality in ALP poisoning cases varied between $70-100 \%$. It was higher in those who took $>2$ tablets and none of patients who took $>3$ tablets survived.

All cases presented with vomiting and abdominal pain. Diarrhea occurred in $38.3 \%$ of cases and $5 \%$ of cases presented with haematemesis. About $11.7 \%$ of cases presented with GCS 14, $55 \%$ with sinus tachycardia, $46.7 \%$ with shock, $68.3 \%$ with tachypnea, $30 \%$ with hypoxemia and $60 \%$ with metabolic acidosis.

This agrees with Goel A and Aggarwal (2007) who reported that, in mild poisoning nausea, repeated vomiting, diarrhea, headache, abdominal discomfort or pain and tachycardia are common clinical features, and these patients usually show recovery. On the other hand, in moderate to severe poisoning, the signs and symptoms of the gastrointestinal, cardiovascular, respiratory, and nervous systems appear initially. Jaiswal et al., (2009) reported also that all ALP intoxicated patients presented with severe metabolic acidosis. According to Gurjar et al., (2011), temporal correlation in ECG changes in ALP poisoning showed that during the initial 3-6 h sinus tachycardia is predominant. Moreover, Navabi et al., (2018) declared that patients mostly die from cardiovascular collapse, refractory shock, 
severe acidemia, fulminant hepatic failure, and/or adult respiratory distress syndrome.

To combat ROS and reduce their damage, living organisms have evolved complex antioxidant systems. The TAC of the system is equal to the amount of endogenous antioxidants (Rani and Mythili, 2014). Measurement of the antioxidant capacity of biological fluids represents an indicator of the overall ability of the body to counteract ROS, prevent oxidative damage and counter oxidative stress-related diseases (Kusano and Ferrari, 2008). While phosphine-induced oxidative damage has been well established in animal studies, there is a lack of evidence regarding measurement of TAC in patients with ALP poisoning (Kariman et al., 2012).

Regarding oxidative stress biomarkers in the current study (Table 3), MDA was significantly increased, and TAC was significantly reduced in ALP poisoned patients at time of admission indicating severe and rapid oxidative stress induced by ALP poisoning in comparison to control healthy individuals who lived in the same environment and pollution (Figure 1 \& 2). Moreover, by assessing levels of MDA and TAC among survivors and expired patients (Table 4), the levels of MDA were significantly high, and levels of TAC were significantly low in patients who died (Figure 3 $\& 4)$. These findings indicated that ALP exerts its toxicity by inducing oxidative stress releasing ROS and compromising antioxidant protection mechanism.

Kariman et al., (2012) also reported that, acute ALP exposure resulted in increased MDA levels and decreased plasma TAC of patients. This result is supported by Gouda et al., (2018) who stated that $\mathrm{PH}_{3}$ leads to enhanced production of ROS and induces oxidative stress through inhibition of antioxidants enzymes e.g., glutathione, glutathione reductase, catalase and superoxide dismutase. These alterations accelerate lipid peroxidation which result in cell membrane injury, ionic barrier destruction, nucleic acid damage and finally cell death.
Moreover, the AUCs for MDA and TAC were higher than the "Hosmer and Lomeshaw" acceptability requirements (Table 5). A cut ${ }^{-}$off value of MDA was $68.0 \mu \mathrm{mol} / \mathrm{L}$ and by operating the ROC curve and regression analysis, any MDA levels above $68.0 \mu \mathrm{mol} / \mathrm{L}$ at the time of admission were associated with poor outcomes with $100 \%$ sensitivity, $97.6 \%$ specificity and $98.3 \%$ accuracy for prediction of mortality (Figure 5). On the other hand, a cut $^{-}$off value of TAC was $0.45 \mathrm{mmol} / \mathrm{L}$ and by operating the ROC curve and regression analysis, any TAC levels below $0.45 \mathrm{mmol} / \mathrm{L}$ at the time of admission were associated with poor outcomes with $100 \%$ sensitivity, $92.7 \%$ specificity and $95 \%$ accuracy for prediction of mortality (Figure 6). This revealed that both MDA and TAC were sensitive in prediction of ALP poisoning mortality at the time of presentation within the first 6 hours of intoxication and the MDA level showed more specificity as well as accuracy for prediction of the poor outcomes.

The limitations of this study were the small sample size in both groups and the indetermination of free radicals amounts along with the oxidative stress biomarkers "MDA and TAC" during the study.

\section{CONCLUSIONS}

The present study suggests the role of severe and rapid oxidative stress pathway in ALP toxicity that could occur early within the $1^{\text {st }} 6$ hours of intoxication. ALP poisoning associated with depletion of endogenous antioxidants and hence weakened antioxidant guard that might be closely related to the rapid death scenarios. Both blood MDA and TAC were highly sensitive in prediction of poor outcomes in ALP poisoning at the time of presentation and can be used as predictors biomarkers of ALP poisoning mortality. Moreover, the MDA level showed more specificity as well as more accuracy for prediction of the poor outcomes in such highly fatal poisoning. 


\section{RECOMMENDATIONS}

The current work could open avenues for future studies to define the potential therapeutic role of early administration of exogenous antioxidant agents with large doses for treatment of acute ALP poisoning.

\section{Acknowledgements}

The authors wish to express their full gratitude to the nurses of "Toxicology Unit of Emergency Hospital, Mansoura University" and the technicians in "Clinical Pathology Department, Mansoura University”.

Declaration of interest:

All authors confirm that there is no organization support for this submitted work and there are no financial connections with any organizations that may have an interest in the present work submitted.

\section{REFERENCES}

1. Agrawal VK, Bansal A, Singh RK, Kumawat BL, Mahajan P (2015): Aluminum phosphide poisoning: Possible role of supportive measures in the absence of specific antidote. India Crit Care Med.;19 (2):109-112.

2. Ayala A, Muñoz MF, Argüelles S (2014): Lipid peroxidation: production, metabolism, and signaling mechanisms of malondialdehyde and 4-hydroxy-2-nonenal. Oxid med cell long.; 360438.

3. Badawi S, Alseidy A, Alfeki A, Mansour M, Abd el-hamid A (2018): Metal phosphide poisoning in Menoufia University Hospitals. Menoufia Med J.; 31, 816-821.

4. Bajpai SR (2010): Aluminium phosphide poisoning: Management and prevention. $J$. India Acad Forensic Med.; 32: 352-354.

5. Bogle RG, Theron P, Brooks P, Dargan PI, Redhead J (2006): Aluminium phosphide poisoning. Emerg Med J.; 23(1): e3.

6. Farahani M V, Soroosh D, Marashi SM (2016): Thoughts on the current management of acute aluminum phosphide toxicity and proposals for therapy: an evidence-based review. India J Crit Care Med.; 20, 724-730.

7. Goel A, Aggarwal P (2007): Pesticide poisoning. Natl Med J India; 20:182-191.
8. Gouda AS, El-nabarawy NA, Ibrahim SF (2018): Moringa oleifera extract (Lam) attenuates aluminium phosphide-induced acute cardiac toxicity in rats. Toxicol Reports; 5, 209212.

9. Gunnell D, Eddleston M, Phillips MR, Konradsen F (2007): The global distribution of fatal pesticide self poisoning: Systemic review. BMC Public Health; 7:357 - 371.

10. Gurjar M, Baronia AK, Azim A, Sharma K (2011): Managing aluminum phosphide poisonings. J Emerg Trauma Shock.; 4(3): 378-384.

11. Hashemi-Domeneh B, Zamani N, Hassanianmoghaddam H, Rahimi M, Shadnia S, Erfantalab P (2016): A review of aluminium phosphide poisoning and a flowchart to treat it. Arh Hig Rada Toksikol.; 67, 183- 193.

12. Jaiswal S, Verma RK, Tewari N (2009): Aluminium phosphide poisoning: Effect of correction of severe metabolic acidosis on patient outcome. India J Crit Care Med.;13 (1):21-24.

13. Kariman H, Heydari K, Fakhri M, Shahrami A, Dolatabadi AA, Mohammadi HA, Gharibi M (2012): Aluminium phosphide poisoning and oxidative stress: serum biomarker assessment. J Med Toxicol. Sep; 8(3):281-4.

14. Khan F, Garg VK, Singh AK (2018): Role of free radicals and certain antioxidants in the management of huntington's disease: a review. J Anal Pharm Res.; 7(4):386-392.

15. Koracevic D, Koracevic G, Djordjevic V (2001): Method for the measurement of antioxidant activity in human fluids. J Clin Pathol; 54:356-61.

16. Kusano C, Ferrari B (2008): Total antioxidant capacity: a biomarker in biomedical and nutritional studies. J Cell Mol Biol; 7: 1-15.

17. Leone $M$, Asfar $P$, Radermacher $P$, Vincent JL, Martin C (2015): Optimizing mean arterial pressure in septic shock: a critical reappraisal of the literature. Crit Care; 19(1): 101.

18. Mathai A, Bhanu M (2010): Acute aluminium phosphide poisoning: Can we predict mortality? India J Anaesth; 54:302 - 307. 
19. Meena MC, Sachin Mittal, Yashoda Rani (2015): Fatal aluminium phosphide poisoning. Interdiscip. Toxicol.; 8(2): 65-67.

20. Mehrpour O, Jafarzadeh M, Abdollahi M (2012). A systematic review of aluminium phosphide poisoning. Arh. Hig. Rada Toksikol:;63:61-73.

21. Nath NS, Bhattacharya I, Tuck AG, Schlipalius DI, Ebert PR (2011): Mechanisms of phosphine toxicity. J Toxicol; 494168.

22. Navabi SM, Navabi J, Aghaei A, Shaahmadi Z, Heydari $R$ (2018): Mortality from aluminum phosphide poisoning in Kermanshah Province, Iran: characteristics and predictive factors. Epidemiol health, 27;40: e2018022.

23. Oghabian Z, Mehrpour O (2016): Treatment of Aluminium Phosphide Poisoning with a Combination of Intravenous Glucagon, Digoxin and Antioxidant Agents. Sultan Qaboos Univ Med J.; 16(3): 352-355.

24. Rani AJ, Mythili SV(2014): Study on total antioxidant status in relation to oxidative stress in type 2 diabetes mellitus. J Clin Diagno Res; 8(3):108-110.

25. Sheta AA, El-Banna AS, Elmeguid RA, Mohamed HE, Gad NH (2019): A study of the predictive factors of mortality in acute poisoning with aluminum phosphide with special reference to echocardiography and SOFA score. Environ Sci Pollut Res Int; 26(32):33135-33145.

26. Singh Y, Joshi S C, Satyawali V, Gupta A (2014): Acute aluminium phosphide poisoning, what is new? Egy J Int Med.; 26(3): 99-103.

27. Yagi K (1984). Assay of blood plasma or serum. Methods Enzymol.;109:328-331. 